\title{
Wind and Weather Facility for Testing Free-Flying Drones
}

\author{
Flavio Noca * \\ HEPIA, HES-SO University of Applied Sciences, Geneva, 1202, Switzerland \\ Guillaume Catry $^{\dagger}$, Nicolas Bosson, Luca J. Bardazzi, Sergio Márquez, and Albéric Gros \\ WindShape, Geneva, 1203, Switzerland
}

\begin{abstract}
Drones will soon fill our aerial ecosystem in the field of imaging/cartography, parcel delivery, and passenger transport, and will need to operate around the clock in arbitrary atmospheric conditions, especially in adverse weather conditions during emergency situations. Drones are much smaller than conventional aircraft and are thus more sensitive to weather conditions. Today, traditional drone testing techniques are of poor quality. Drones are either tested outdoors quite remote from the observer, in not well documented, uncontrolled and unpredictable weather conditions, or tightly strapped onto a support in a conventional wind tunnel with laminar and uniform wind flows, which are inadequate representations of atmospheric conditions relevant to drones. Existing tests, thus, entail a high risk of reaching false conclusions about drone performance. In order to resolve the issues associated with traditional wind tunnels or outdoor testing protocols, a real weather simulator for testing flying vehicles in various and controllable atmospheric conditions was developed. The wind and weather facility consists of an array of a large number of fans that may be arranged in various patterns on demand. It subjects drones to winds of variable intensity and direction as well as various weather conditions (such as rain, snow, hail, fog etc.) that reflect real world situations. These tests can rate drones according to their capacity in maintaining a proper flight attitude and tackling flight perturbations in an urban, countryside, or high altitude environment.
\end{abstract}

\section{Introduction}

$\mathrm{D}$ RONES are aerial vehicles that fly without an onboard pilot. In the past, the terminology drone was restricted to military platforms. The word drone is today accepted worldwide to refer to both military and civilian systems. If they are unmanned, they are classified officially by the FAA (Federal Aviation Administration) and the ICAO (International Civil Aviation Organization) as Unmanned Aircraft Systems or UAS (which also include associated ground systems), although they are also commonly called Unmanned Aerial Vehicles (UAV). If they carry a handful of passengers, drones are known as Urban Air Mobility (UAM) aircrafts or drone-taxis (although no official label yet exists since these vehicles are still experimental). UAVs are used nowadays for multiple applications, from recreational to industrial and commercial activities such as mapping/cartography/inspection and delivery. In turn, UAMs are expected to thrive in the coming decade through the efforts of traditional aircraft or helicopter manufacturers as well as a number of burgeoning startups.

Drones can be remotely piloted (as in traditional recreational radio-controlled flyers), but the trend is towards fully autonomous systems, whether for unmanned or manned drones. However, such automation entails a number of risks and challenges that need to be mitigated. As an example, we note that on January 25, 2019, a Matternet drone operated by Swiss Post and carrying blood samples fell into Zürich Lake.

Similarly to the aircraft industry, the performance of drones needs to be assessed in order to minimize the occurrence of such events in the future. Above a certain size or weight (yet to be standardized), drones will be certified to fly following traditional aircraft procedures (which will need to be amended for the autonomous aspect). However, for smaller size drones (including single passenger drone-taxis), certification procedures will need to be greatly revised since these vehicles are extremely prone to weather conditions, including gusts and shear. Paradoxically, it is in bad weather, when traditional mobility channels, such as roads, are unusable, that drones would be critical, in particular for emergency and safety operations.

Nowadays, it is natural for most drone manufacturers to run operational demonstrators or obtain waivers for specific

*Professor, Center for Hydro \& Aero, Rue de la Prairie 4; flavio.noca@hesge.ch; http://www. chachacha.ch

${ }^{\dagger} \mathrm{CEO}$, Rue de Bourgogne 25; guillaume.catry@ windshape.ch; http://www.windshape.ch 


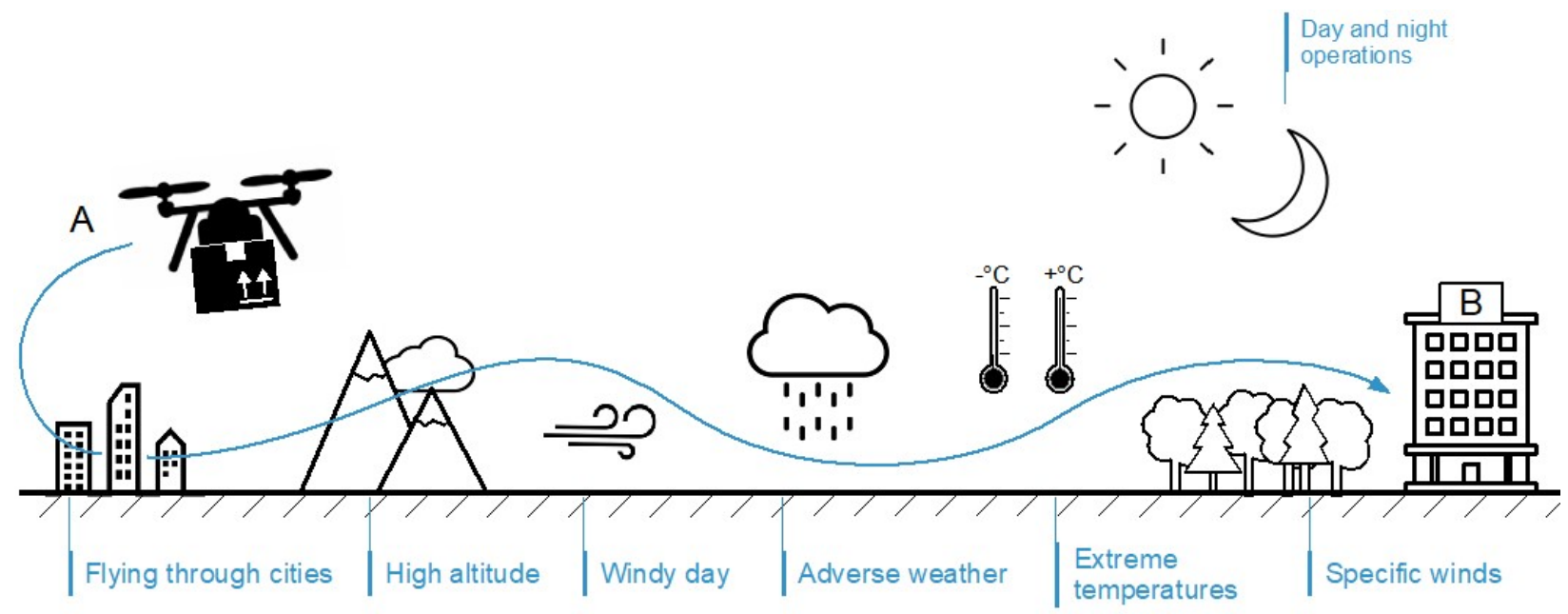

Fig. 1 Drone airworthiness will have to be properly tested and rated for realistic scenarios, and in particular, adverse weather conditions.

operations but in the near future this industry will need to rely on performance and safety standards. Such standards will facilitate the development of drones and reduce the time to market.

Various international regulatory agencies (in particular, JARUS - Joint Authorities for Rulemaking on Unmanned Systems ${ }^{*}$ ) are in the process of setting up regulations for the safe operation of drones, i.e. SORA (Specific Operations Risk Assessment). It is for the good of the industry that drones will have to pass official testing procedure (as it is the case today for traditional transportation systems), and will be rated according to their ability to tackle realistic scenarios (Figure 1), and in particular, adverse weather conditions.

\section{Traditional testing facilities}

In the last century, wind tunnels were pioneered by Eiffel [1] in 1908 and Prandtl [2] in 1909 to support the development of the burgeoning aircraft industry. These test facilities were intended for aircrafts, which either fly in relatively quiet atmospheric conditions (for example at 30’000 ft flight levels) or are so large and heavy that they are largely unaffected by turbulence scales that are small relatively to the size of the craft. However, such wind tunnels are inadequate for drones. Laminar, low turbulence, steady, and flat-profile winds are not representative of atmospheric

Http://www.jarus-rpas.org/

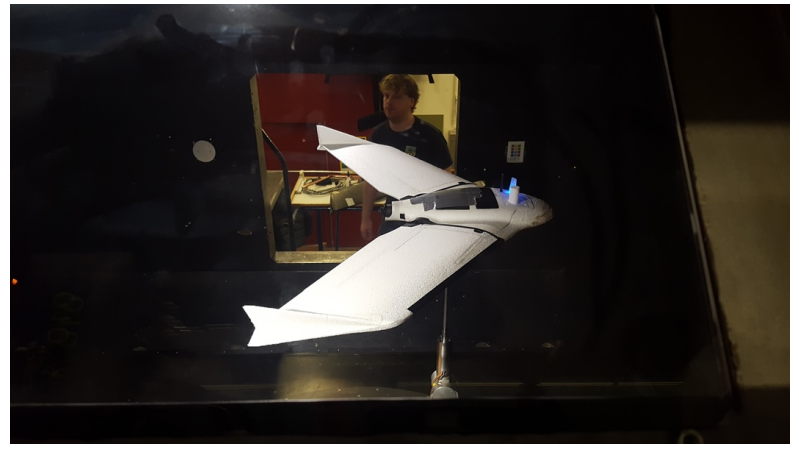

(a)

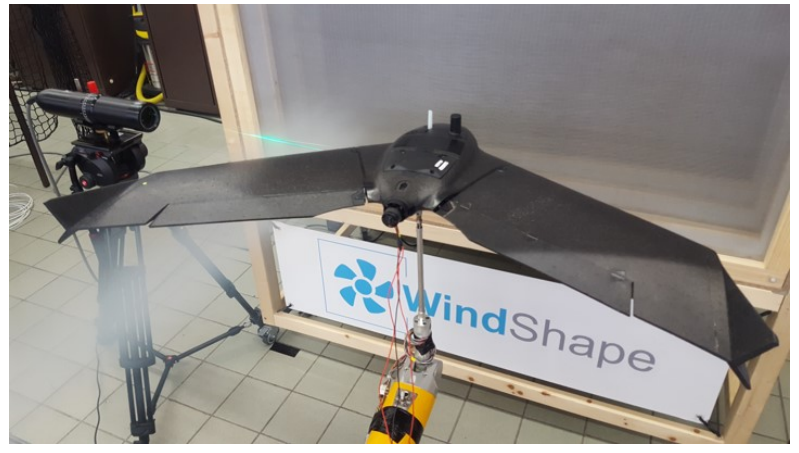

(b)

Fig. 2 Aerodynamic development of senseFly eBeeX in a traditional wind facility setup (a) at the ETH Zürich wind tunnel, and (b) in a WindShape facility used as a traditional wind facility, with uniform, low turbulence flow (with screens and honeycombs) and the drone tightly attached to a robotic arm. 
conditions encountered by small flying vehicles. In such tunnels, spatial and temporal wind gradients can only be produced with added perturbations, either passive (grids, roughness elements) or active (vibrating blades) [3]. These flow obstructions offer little control, only reproduce time-averaged statistics of atmospheric turbulence, or generate overly simplified canonical flows, such as periodic modulations.

Aircraft models or full-scale drones are generally tethered to a rigid support or a sting (Figure 2), and cannot move/fly freely in the flow, especially because of the confining tunnel walls. Drones have been flown freely in large wind tunnels [4] as well as climatic facilities [5-7], although the exact flow structure at the instantaneous drone position is generally unknown (unless the flow is steady).

Currently, there are no solutions for rating drones and single-passenger aircraft in well-defined wind/weather configurations. Due to the lack of testing solutions, manufacturers have no choice other than testing their flying vehicles in the outdoors. However, this testing methodology suffers from many drawbacks, including poor accuracy, lack of reproductibility, dependence on day-by-day weather forecast, unknown and non-controllable flow conditions, short test times, large distance between drone and tester. Under such conditions it is difficult to assess and evaluate drone aerodynamics and provide robust measures of their airworthiness.

\section{WindShape technology}

\section{A. General description}

In order to resolve the issues associated with traditional wind tunnels or outdoor testing protocols, we have developed a real wind and weather simulator [8] for testing flying vehicles in various and controllable atmospheric conditions, including arbitrary wind speed, temperature and direction (even vertical flow for simulating landing/descent configurations), as well as turbulence, hail, rain, snow, and sandstorms. The novel wind facility allows free-flight maneuvering, is completely modular, can be assembled in any desired geometry, and can be made as large as desired while maintaining a small footprint. It is capable of generating gusts (temporally varying winds) and arbitrary wind profiles (shear flows) in any direction. Foremost, the flyer is always at hands-reach from the tester while performing actual flight maneuvers. A motion tracking system (motion capture cameras or mocap) is integrated into the facility in order to measure the drone position and attitude (Figure 3).

The product has been designed and is being commercialized by a Swiss company, WindShape The first such facility (Figure 4) was purchased by the Aeronautics Department at the California Institute of Technology (Caltech) and inaugurated on October 24, 2017, within its new Center for Autonomous Systems and Technologies (CAST)

\section{B. Multiple-fan technology}

The wind facility is based on a multiple-fan technology, which is not novel in itself. A number of conventional tunnels use multiple-fan, although they are made to rotate at the same speed (such as in the National Full-Scale

\footnotetext{
http://www.windshape.ch

http://cast.caltech.edu
}

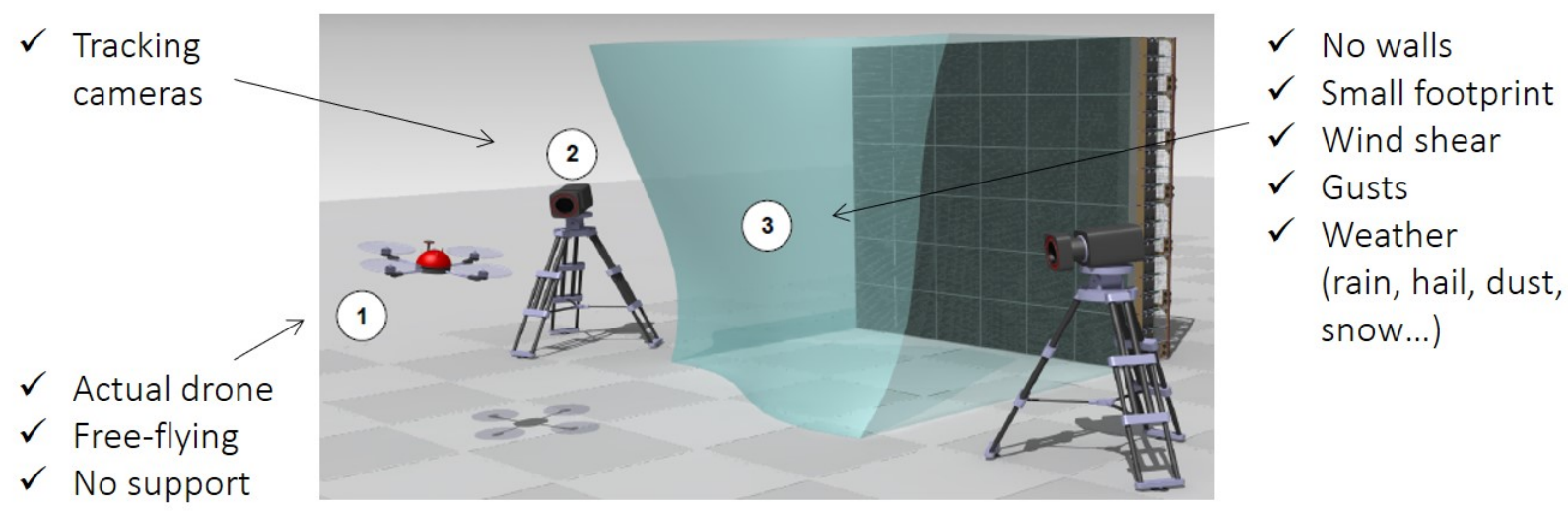

Fig. 3 Wind facility for drone-testing based on multiple-fan technology [8]. 


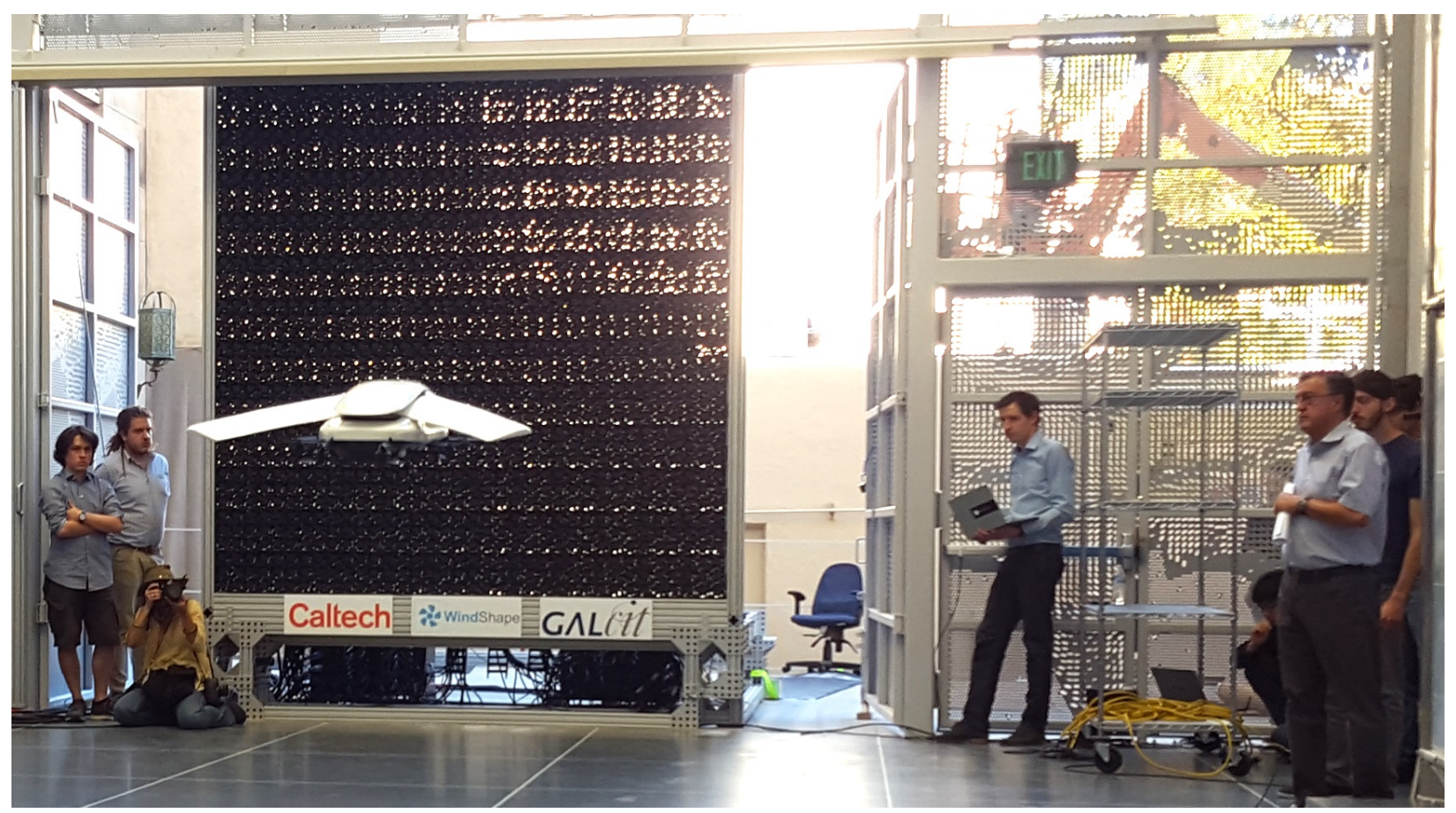

Fig. 4 The first UAV flight research and test facility was built by Swiss company WindShape for the Aeronautics Department at the California Institute of Technology (Caltech) and inaugurated on October 24, 2017, within its new Center for Autonomous Systems and Technologies (CAST, http://cast. caltech. edu).

Aerodynamics Complex at NASA Ames Research Center, Moffet Field, CA, USA). Atmospheric boundary layers have been simulated with arrays of individually-controlled jets [9] and fans [10-12]. Fan arrays have been used to generate gusts and shears around pliable structures [13, 14] or micro-air vehicles (MAVs) [15]. One hundred years ago, the single-fan technology used by Eiffel and Prandtl was not novel either (the Wright brothers and others had experimented with similar devices), but the tunnels they designed were a unique and innovative tool for the aircraft industry. Similarly, WindShape technology is distinctive as it addresses the needs of the drone industry. In particular, the patented modular fan system [8] enables the stacking of an unlimited number of fans (1'296 for the Caltech facility) for high resolution and fast response, as well as arbitrary wind-generating geometries that can be modified at will and over time.

\section{Wind pixels}

The technology consists of an array of a large number of fans (approximately 150 fans per square meter) stacked in arbitrary fashion. Each single fan can be controlled independently and can, thus, be assimilated to a wind pixel (wpx). WindShape wind walls are composed of a great number of wind pixels (12.5 wpx/m in the Caltech configuration). This feature allows fine control over the generated wind properties, which in traditional tunnels requires extra flow management devices (proper nozzle geometry, flow control devices, vanes etc.). In addition, the low inertia of small-size fans enables fast changes in wind speed. Gusts of wind and shear flows can be faithfully reproduced. Laminar wind conditions, with a turbulence intensity below $1 \%$, can be achieved by adding screens and honeycombs in front of the fans (Figure 2), while preserving the independent character of every single wind pixel. Alternatively, traditional wind testing can be performed by placing the model on the aspiration side and by integrating appropriate tunnel walls.

\section{Wind modules}

The basis of the product is what we call a wind module - a wind generation unit composed of nine small fans (Figure 5a, acting like a building brick or $\mathrm{Lego}^{\mathrm{TM}}$ block. These modules are designed to be assembled manually into an array to shape surfaces of fans (Figure 5b). These modules can be arranged onto surfaces of any shapes (Figure 5c). One current version of the module has a square section of $0.058 \mathrm{~m}^{2}$, can generate winds up to $16 \mathrm{~m} / \mathrm{s}$, and requires about $1.1 \mathrm{~kW}$ of electrical power at maximum output. 


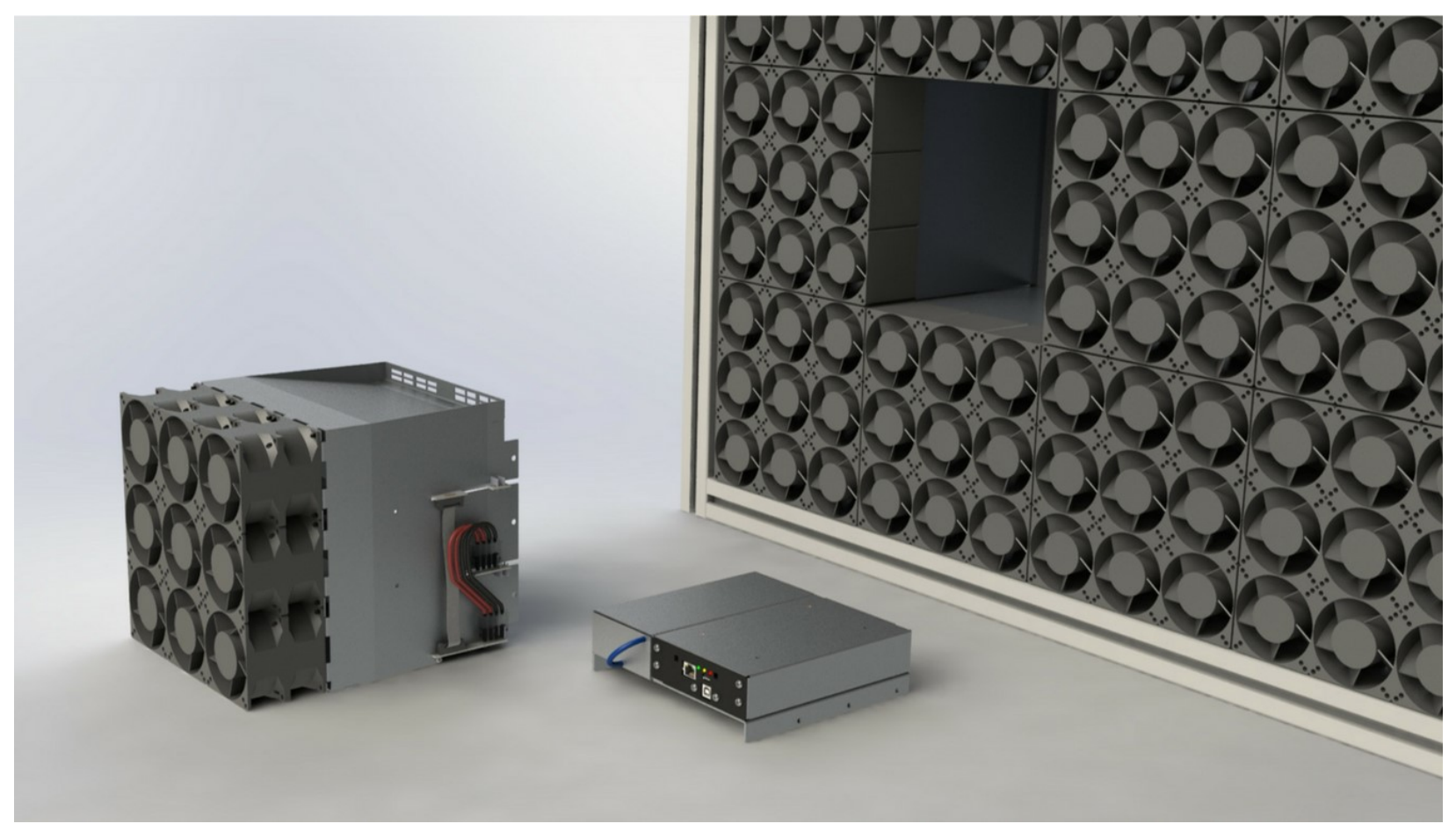

(a)

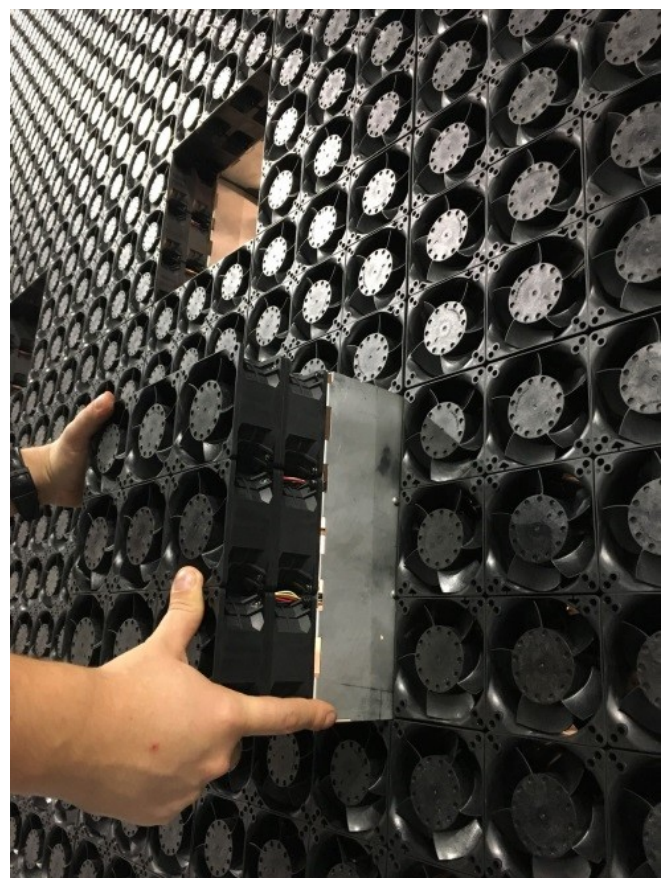

(b)
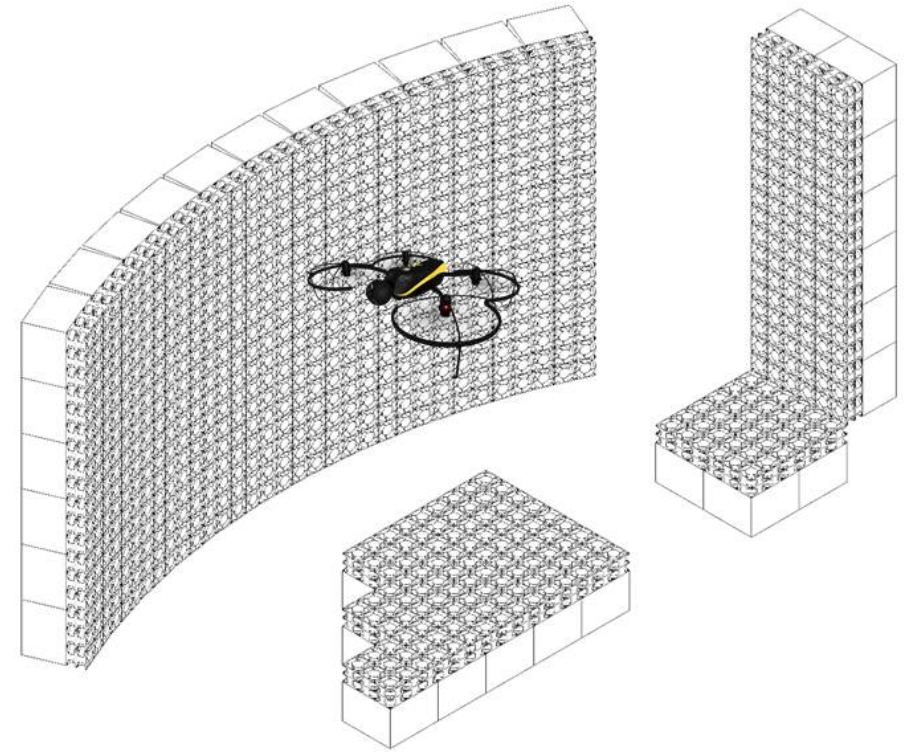

(c)

Fig. 5 (a) The basis of WindShape technology is what we call a wind module - a wind generation unit composed of nine small fans with integrated power, which acts like a building brick or Lego ${ }^{\mathrm{TM}}$ block. (b) These modules can be rapidly assembled into an array to shape surfaces of fans. (c) Wind modules can be stacked into arbitrary shapes and sizes, thus enabling the testing of drones of various dimensions, from small UAVs up to drone-taxis, in arbitrary wind configurations (cruise flight, descent, cross-winds etc.). 


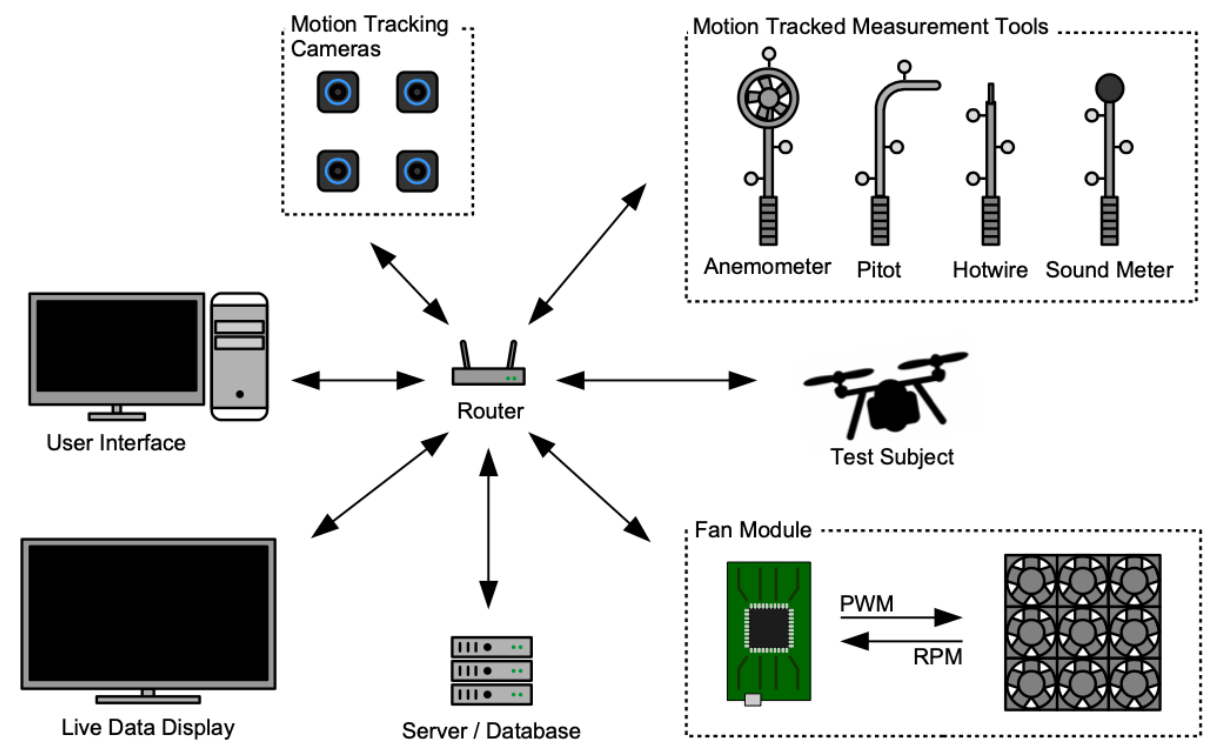

Fig. 6 All elements in the test environment, namely the wind modules, the drone, the sensors, the motion tracking camera system and the user interface are interconnected and share data through a network. The server stores every piece of data in a database and turns it into valuable test information.

The facility being highly modular, thus, lends itself to an unlimited variety of wind configurations, spatially or temporally (Figure 5b):

- A wind wall can be enlarged simply by adding more modules to the wall;

- By moving the modules around, one can change the aspect of the wall (rectangular, square, etc.);

- The wall orientation can be easily modified: for instance, one can recreate the apparent wind of the descent (landing) phase of a multirotor by choosing to place the wind modules in a horizontal plane.

\section{E. System Architecture}

The present technology is also unique in the sense that all elements in the test environment are interconnected through a network (Figure 6. The drone, or more generally the test subject, communicates and can even be controlled through this network. While the drone is in free-flight in the test section, its position and attitude are being captured by the motion tracking camera system and shared on the network. The wind modules are also controlled through this network. The drone can be controlled according to the wind profile or, alternatively, the wind profile can be adjusted based on the drone position.

Other elements, such as flow measurement sensors, can be connected to the network as well. Thus, the measured data are processed directly by the server, which can adapt the flow speed or other flow characteristics accordingly. Moreover, equipping each sensors with markers allows the software to associate each data point with a probe position and orientation.

Finally, while recorded data are paramount for post-processing, it is also crucial to have a live feedback of the test parameters. Having all data centralized in a single database make it possible to have live data visualization (Figure 11].

\section{Drone Testing and Use Cases}

\section{A. Generalities}

A high-speed camera can capture critical information (for instance, propeller vibrations) that is not available with outdoor testing protocols.

Unsteady aerodynamic forces can be extracted from onboard sensors (accelerometer, gyroscope, etc) and the drone trajectory (obtained from the motion tracking cameras). 


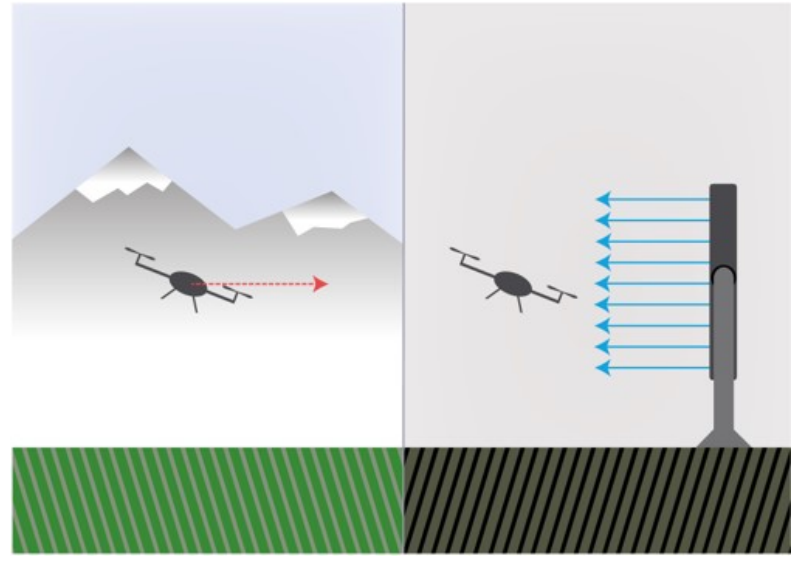

(a)

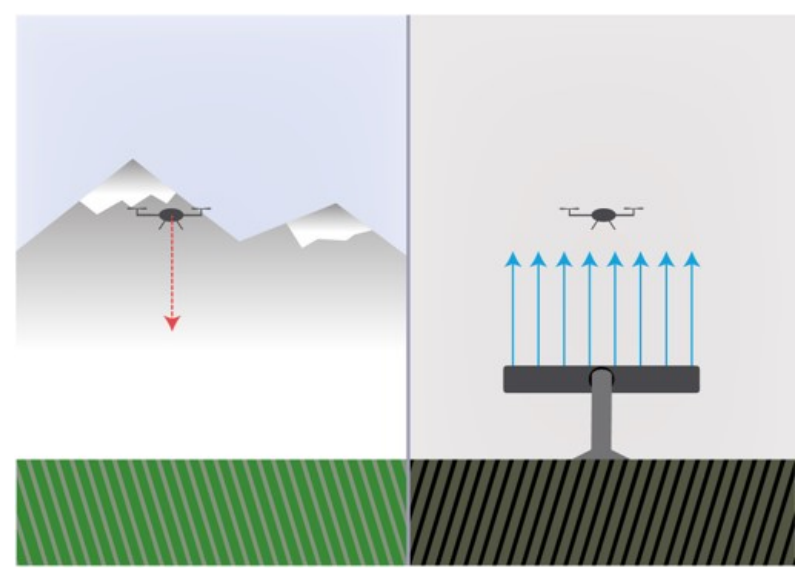

(c)

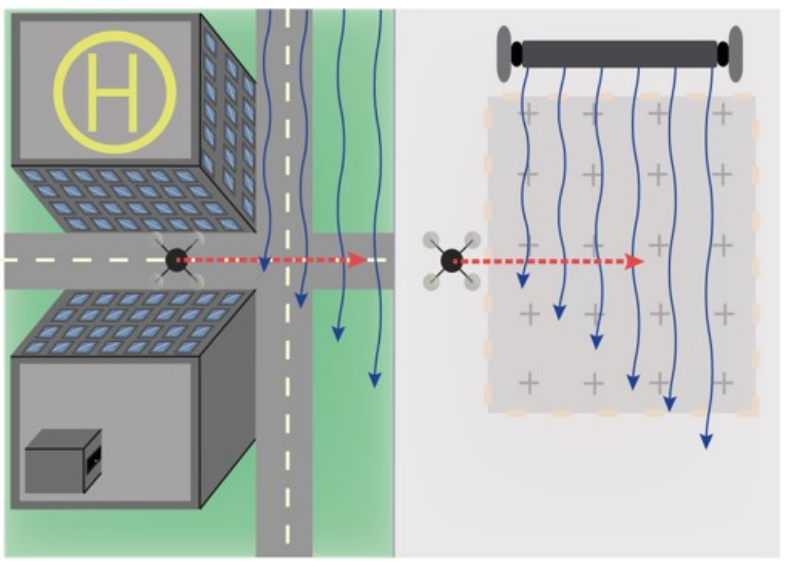

(e)

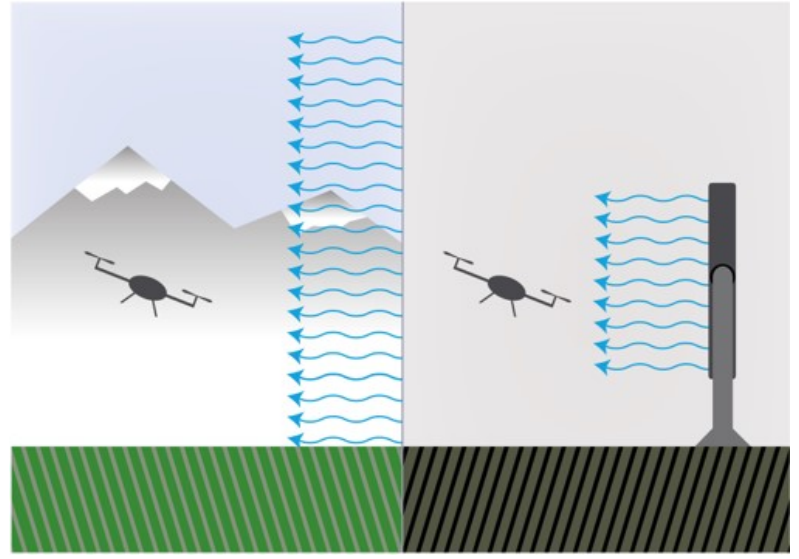

(b)

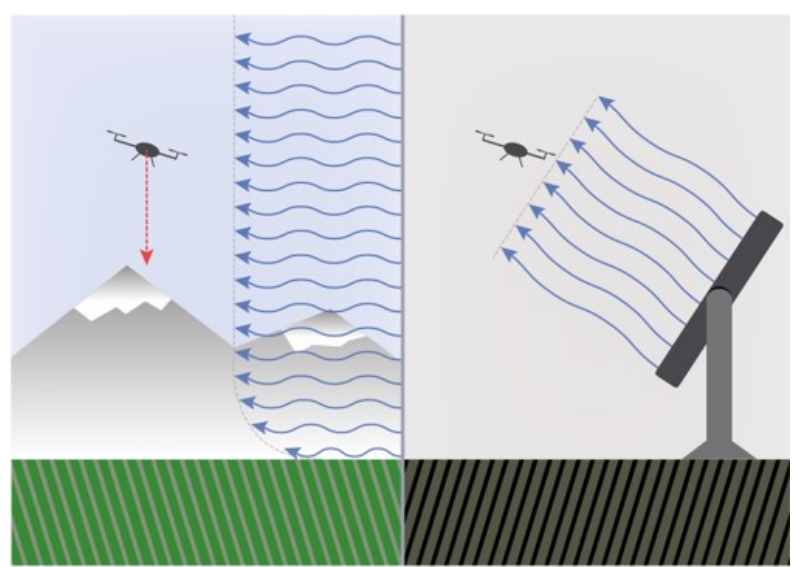

(d)
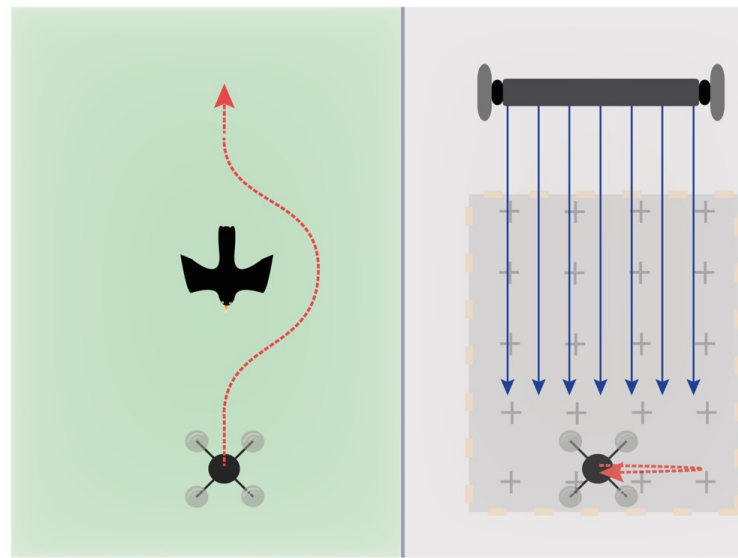

(f)

Fig. 7 UAVs and UAMs can be made to fly freely in a variety of flow configurations, for testing, development, or performance assessment and rating. 


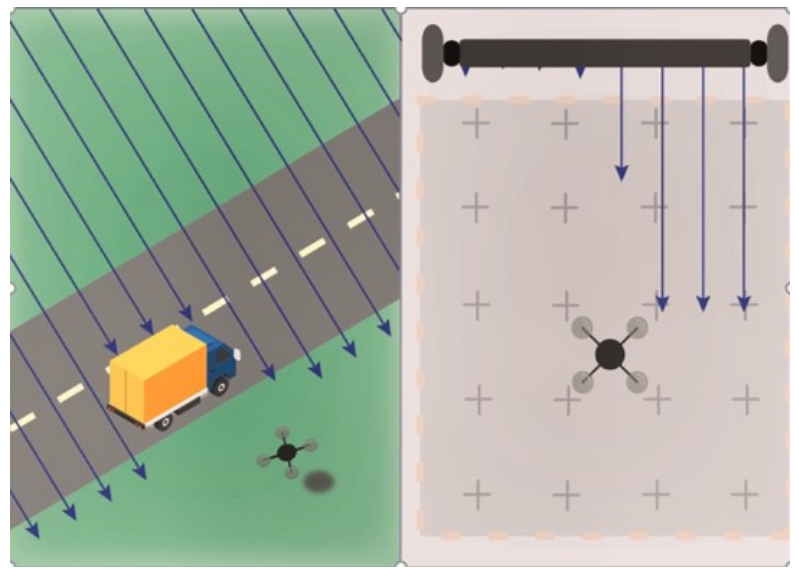

(a)

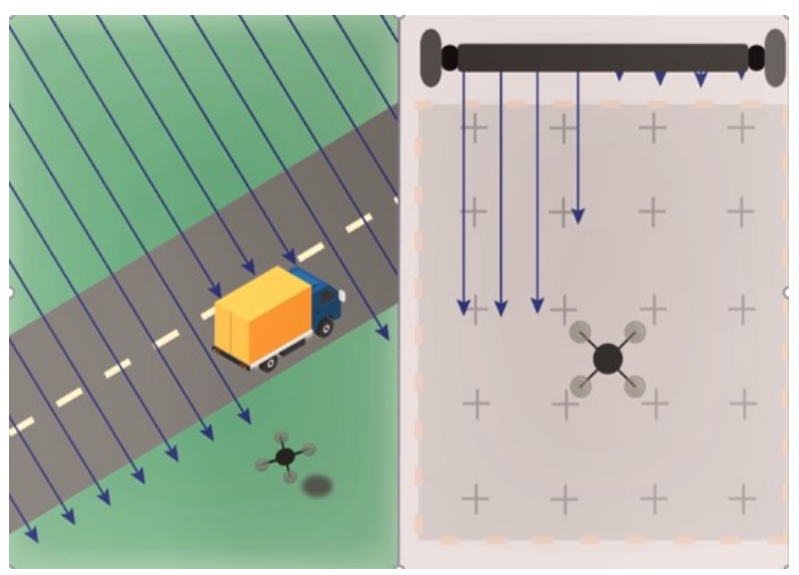

(b)

Fig. 8 UAVs and UAMs can be rated according to their ability in maintaining a stable position in adverse winds.

Flow fields around the craft or its propulsion unit can be quantitatively mapped with standard fluid mechanics measurement techniques such as Particle Image Velocimetry (PIV), Laser Doppler Velocimetry (LDV), or multi-hole pressure probes.

\section{B. Sample of Testing Protocols}

The present wind and weather facilities offer new paradigms for testing autonomous and personal flying systems. In particular, the crafts fly freely (as in their natural environment) in customizable wind configurations. This new testing method offers a wide spectrum of possibilities:

- A variety of wind profiles can be setup, from relative wind (Figure 7a to actual turbulent wind (Figure 7b);

- The facility can be tilted into a horizontal fan array to simulate landing (vertical apparent wind) as depicted in Figure $7 \mathrm{c}$.

- Shear flows, as encountered in urban canyon flows (Figure 7e), can be easily generated with a multiple-fan technology;

- Drones can fly freely without any risk of collision (since the facility has no bounding walls as in conventional tunnels) and can, for instance, be assessed for collision avoidance capability (Figure 7f);

- The technology allows fast changes in wind speed and, thus, the generation of gusts (Figure 8);

- Weather (rain, snow, dust etc.) can be easily inserted as the configuration is open (Figure 97;

- The facility can also be converted into a traditional low-turbulence tunnel for conventional testing with suitable use of screens/honeycombs (Figure2).

\section{Wind and Gusts Resistance}

As drones can fly freely in the flow generated by a multiple-fan wind facility, their ability to withstand strong winds and gusts can be accurately assessed. Figure 11 a depicts a test where the drone is being tracked by motion capture cameras while being subjected to space and time varying flows. The graphs of Figure $11 \mathrm{~b}$ show respectively the wind speed and the drone displacement along the flow direction. Such tests are, for example, conducted to fine-tune flight controllers, or to evaluate the structural resistance in strong winds.

\section{Rain Tests}

To assess and improve drones ability to operate in heavy weather conditions, tests combining rain and wind are conducted in a controlled environment, as shown on Figure $9 \mathrm{~b}$ As the present wind and weather facilities feature open test sections, various elements can be seeded into the flow, such as rain, snow, or sand. The impact of droplet diameter and speed on the drone behavior is currently being investigated, in order to better understand the challenges that need to be addressed. 


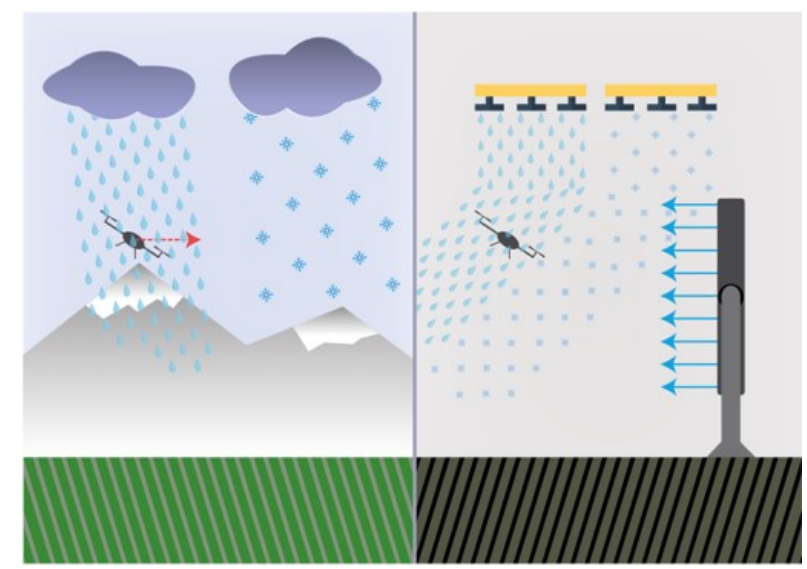

(a)

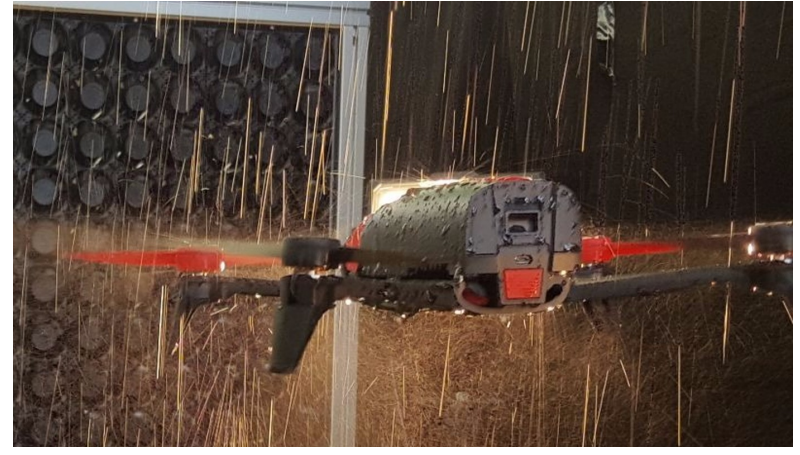

(b)

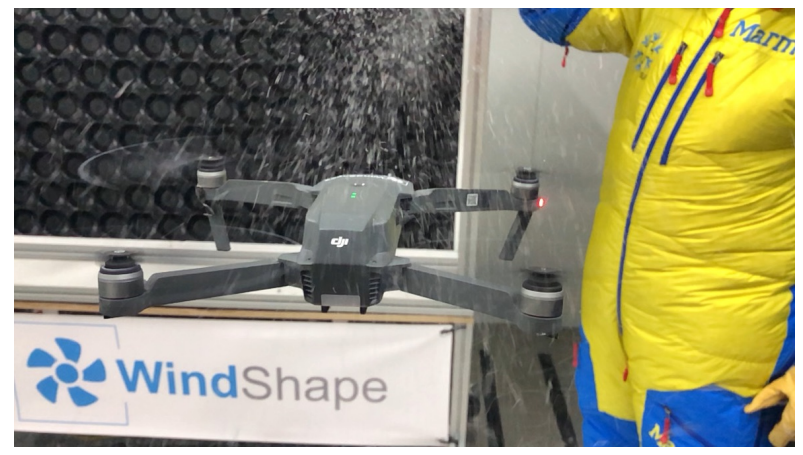

(c)

Fig. 9 (a) The open architecture facilitates the integration of arbitrary weather phenomena, such as (b) rain, (c) snow, dust, hail etc. The small footprint of the facility also allows its integration in existing climatic chambers or wind tunnels (drone in (c) being tested in snowy and windy conditions inside a climatic chamber at the Swiss Institute for Snow and Avalanche Research).

\section{E. Snow}

Snow tests were conducted in a cold chamber provided by WSL Swiss Institute for Snow and Avalanche Research \$ A WindShape facility with a test section of $1.5 \mathrm{~m}$ by $0.75 \mathrm{~m}$ is set up in this chamber, thus allowing for drone testing in freezing, windy and snowy conditions. WSL has developed a unique dendritic-snow production device, which is able to produce nature-identical snow at any time of year. The real-time process of icing at temperatures down to $-20^{\circ} \mathrm{C}$ in snowy conditions is being investigated in collaboration with WSL scientists. Preliminary tests were conducted on a commercial drone in September 2018 (Figure 9c).

\section{Development Perspectives}

\section{A. Variable shapes}

If current fan arrays wind generators can produce an extremely wide range of flows, even richer wind morphologies can be generated by changing the shape of the fan wall itself, or by combining multiple walls.

For instance, an arbitrary multiple-wall geometrical architecture (such as a cube) can be created in order to blow in more than one direction, i.e. horizontally and vertically (to simulate cross-flows for instance).

Moreover, modules don't have to be stacked onto a flat wall: they can be arranged into arbitrary morphologies such as curved walls to produce a dome-like configuration. A curved geometry will enable a 3D wind simulator in time and space. Drones are highly dynamic and are able to change trajectory rapidly thanks to their low inertia. With a

$\sqrt[8]{\text { WwW.slf.ch }}$ 

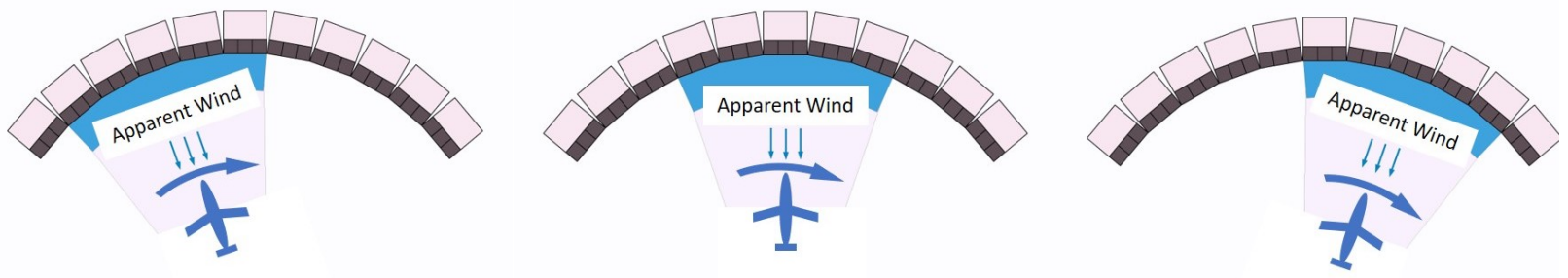

Fig. 10 With modules arranged in a curved geometry, relative wind can be reproduced during a turning maneuver.

curved wall, it is possible to reproduce the change in apparent wind direction resulting from the unsteady maneuvers and changing trajectories (Figure 10p.

\section{B. On the way to UAV and UAM rating and certification}

The drone market has the potential to grow very quickly and one can sense that the lack of regulation is currently preventing this from happening. Hundreds of exciting and promising drone applications in various domains such as air delivery, surveying, agriculture, architecture, security or video entertainment are being developed. Unfortunately, most of them are not viable today because they fall out of the regulation frame.

In a near future, once drone regulations will be established, every flying system wishing to operate in the public airspace will have to be granted certification through relevant test procedures, as it is the case today for existing transportation systems. For instance drones could be rated for water-proofness in a free-flight rain test (Figure 9b), certified for operations in snowy conditions through a cold chamber flight test protocol (Figure 9c) or rated for gust resistance when submitted to a time-varying wind profile (Figure 11). One remaining challenge will be to measure and catalog urban wind environments - and ultimately reproduce them with WindShape technology.

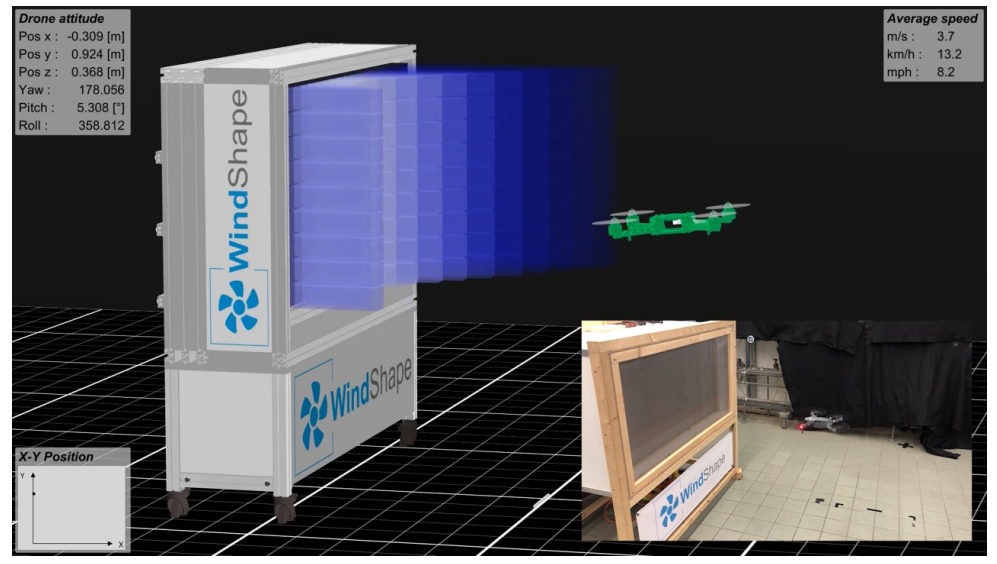

(a)
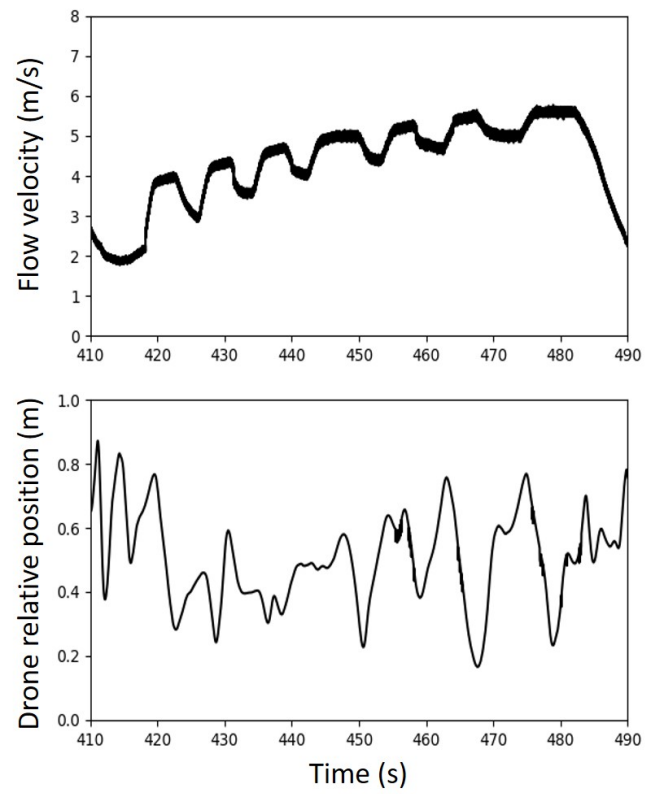

(b)

Fig. 11 (a) As a drone flies freely in a time- and spatially-varying wind, motion tracking cameras measure the position and attitude of the flyer; (b) the ability of the drone in staying within an acceptable range in a fluctuating flow field is an assessment of its performance in a gusty environment (the graphs are for uniform gusts; the drone position is measured along the gust direction). 


\section{Conclusion}

As the needs of each UAV and UAM developer are different, WindShape has come up with a solution that is highly modular, scalable to arbitrary size, and lends itself to an unlimited number of configurations. We have created a real weather simulator for testing flying vehicles in various and controllable atmospheric conditions, including arbitrary wind speed, temperature and direction, as well as turbulence, hail, rain, snow, and sandstorms. The novel wind facility allows free-flight maneuvering in arbitrary gusts and shear flows. Foremost, the flyer is always at hands-reach from the tester while performing actual flight maneuvers. A motion tracking system is integrated into the facility in order to measure the drone position and attitude. Multiple-fan facilities can play an important role for product testing, development, rating, and certification.

\section{Appendix}

On a windy summer day in 2015, while on a beach in Palau (Sardinia, Italy), the first author (Flavio Noca) was watching seagulls hover through turbulent flowfields and maintain their position with respect to the ground and the observer. He then realized that drones ought to be tested in a similar way. They had to be placed in a free-flight and stationary configuration within an arbitrary flowfield, simulating relative winds or actual atmospheric/urban flows. This was contrary to accepted aerodynamic testing dogma, according to which aircraft models had to be strung onto a rigid support and be placed in a laminar, low-turbulence, steady air stream. In the fall of 2015, he instructed his assistant (co-author Guillaume Catry) to stop working on an ongoing drone project and instead focus on the design of a test facility to assess the performance of drones. The basis of the facility, a multiple fan system, was inspired by a small wind tunnel that had been built by Morteza Gharib and his students at Caltech in the summer of 2011 (https://www.caltech.edu/about/news/fan-caltech-1960) to study the interaction of gusts and shear with pliable structures [13, 14]. The facility drew inspiration from a computer-fan based wind tunnel at Oklahama State University (OSU) for testing micro-air vehicles [15]. The OSU publication [15] cites two previous computer-fan setups used as low-cost demonstration wind tunnels, one at the Strategic Air Command Museum and the other at the University of Kentucky. When Noca met his former PhD co-advisor (Gharib) at an American Physical Society meeting in November 2015, he learned about an ongoing project for a Center for Autonomous Systems and Technology (CAST) at Caltech. Gharib asked for any "good" ideas that could be implemented within the center, and Noca suggested the project that Catry was working on: a large-scale multiple-fan facility for testing free-flying drones. WindShape was founded a few months later in order to design and manufacture the facility at Caltech. The project was made possible by the patented modular design, which enabled a simple way of assembling an unlimited number of fans. "WindShaper 1" was inaugurated on October 24, 2017.

\section{Acknowledgments}

This work has received support from HEPIA - HES-SO University of Applied Sciences; the Swiss Confederation; the Canton of Vaud; the Canton of Geneva; the Fondation pour l'Innovation Technologique (FIT); Optitrack by NaturalPoint; Swissnex Network; House of Switzerland (HoS); Genilem; Platinn; Procter \& Gamble.

\section{References}

[1] Eiffel, G., La résistance de l'air et l'aviation - Expériences effectuées au Champ-de-Mars, Dunod, Paris, 1910.

[2] Prandtl, L., Gottingen wind tunnel for testing aircraft models, NACA TN 66, Washington, 1920.

[3] Cermak, J. E., "Progress in physical modeling for wind engineering," Journal of Wind Engineering and Industrial Aerodynamics, Vol. 54-55, 1995, pp. $439-455$.

[4] Mohamed, A., Abdulrahim, M., Watkins, S., and Clothier, R., "Development and Flight Testing of a Turbulence Mitigation System for Micro Air Vehicles,” Journal of Field Robotics, Vol. 33, No. 5, 2016, pp. 639-660.

[5] Automotive Center for Excellence (ACE), University of Ontario Institute of Technology, Canada.

[6] Jules Verne Climatic Wind Tunnel, Scientific and Technical Center for Building (CSTB), Nantes, France.

[7] Wind Engineering, Energy and Environment Research Institute (WindEEE), Western University, Ontario, Canada.

[8] Noca, F., and Catry, G., "Wind Generation Means and Wind Test Facility Comprising the Same," PCT/EP2017/064451, 2016. 
[9] Teunissen, H. W., "Simulation of the planetary boundary layer in a multiple-jet wind tunnel," Atmospheric Environment (1967), Vol. 9, No. 2, 1975, pp. 145 - 174.

[10] Nishi, A., and Miyagi, H., “A computer-controlled wind tunnel," Journal of Wind Engineering and Industrial Aerodynamics, Vol. 54, 1995, p. 493.

[11] Smith, J., Masters, F., Liu, Z., and Reinhold, T., "A simplified approach to simulate prescribed boundary layer flow conditions in a multiple controlled fan wind tunnel," Journal of Wind Engineering and Industrial Aerodynamics, Vol. 109, 2012 , pp. 79 - 88.

[12] Wang, J.-Y., Meng, Q.-H., Luo, B., and Zeng, M., "A multiple-fan active control wind tunnel for outdoor wind speed and direction simulation,” Review of Scientific Instruments, Vol. 89, 2018, pp. 837-846.

[13] Kim, D., Cossé, J., Huertas Cerdeira, C., and Gharib, M., "Flapping dynamics of an inverted flag," Journal of Fluid Mechanics, Vol. 736, 2013, p. R1.

[14] Cossé, J. T., "On the Behavior of Pliable Plate Dynamics in Wind: Application to Vertical Axis Wind Turbines," Ph.D. thesis, California Institute of Technology, 2014.

[15] Johnson, E., and Jacob, J., "Development and Testing of a Gust and Shear Wind Tunnel for NAVs and MAVs," 47th AIAA Aerospace Sciences Meeting including The New Horizons Forum and Aerospace Exposition, 2009. 